\section{MS12-P2 Crystallization of transcriptional metalloregulator protein CueR in complex with $\mathrm{Hg}^{2+}$}

Valeria Bugris $^{1}$, Ria K. Balogh ${ }^{2}$, Veronika Harmath ${ }^{1}$, Zsolt Filákovics ${ }^{1}$, Sándor Brockhauser ${ }^{1}$, Attila Jancsó ${ }^{2}$

1. Biological Research Center, Hungarian Academy of Sciences, Temesvári krt. 62, H-6726 Szeged, Hungary

2. University of Szeged, Dóm tér 7, H-6720 Szeged, Hungary

email: bugris.valeria@brc.mta.hu

The optimal concentration of metal ions in bacteria is controlled by transcriptional metalloregulators. MerR proteins operate by an activation mechanism based on a conformational change of the DNA-bound protein upon metal ion coordination. This also affects the structure of the DNA and therefore, RNA polymerase can initiate the transcription of the regulated genes leading to the formation of proteins the role of which is the removal of metal ion from the cell. The MerR family member CueR (Cu efflux Regulator) protein regulates the intracellular amount of $\mathrm{Cu}$-ion in several strains of bacteria. The CueR protein gives a transcriptional response only for singly charged transition metal ions $\left(\mathrm{Cu}^{\mathrm{I}}, \mathrm{Ag}^{\mathrm{I}}\right.$ and $\left.\mathrm{Au}^{\mathrm{I}}\right)$. Our aim is to solve the structure of the CueR protein in complex with an unfavoured divalent metal ion, the strong binder $\mathrm{Hg}^{\mathrm{II}}$. From these studies we will learn about the structural differences compared with the already available protein structures containing monovalent metal ions [Changela et al, Science, 2003, 301: 1383]. The main goal of these studies is to understand the structural details of bacterial metal ion regulatory mechanisms on molecular basis. For this purpose we have optimized the conditions of the CueR protein expression and purification in $E$. coli, and set up a crystallization screen with similar conditions as described in the literature. The results of these experiments will be presented.

*R.K. Balogh and V. Bugris contributed equally to this work.

Keywords: crystallyzation, CueR, complex with $\mathrm{Hg}(\mathrm{II})$

\section{MS12-P3 The latest developments of detecting and sorting protein micro- and nano-crystals}

Lars Thormann ${ }^{1}$, Robin Schubert ${ }^{1}$, Arne Meyer ${ }^{2}$, Karsten Dierks ${ }^{2}$, Markus Perbandt ${ }^{1}$, Christian Betzel ${ }^{1}$

1. University of Hamburg, Center of Ultrafast Imaging, Laboratory for Structural Biology of Infection and Inflammation, c/o DESY, Build. 22a Notkestr. 8522607 Hamburg / Germany

2. Xtal Concepts GmbH, Marlowring 19, 22525 Hamburg / Germany

email: lars.thormann@chemie.uni-hamburg.de

Applying high brilliant Synchrotron Radiation X-ray free-electron laser (XFELs) radiation in combination with Serial Femto Second Crystallography (SFX) offers today the possibility to collect diffraction data from micro- and nano-sized protein crystals at room temperature before radiation damage will be effective. Due to the superior intensity of FEL radiation the required size of the protein crystals to be used for SFX is significantly smaller compared to those used for conventional diffraction data collection. However, in comparison to conventional protein crystallography a high number of these crystals is required $\left(>10^{9}\right)$, delivered in concentrated suspensions via a particular sample delivery jet technology. Exceptionally some large crystals or particles in the suspension can unfortunately block the sample delivery jet and can cause problems along the SFX data collection. Therefore in different approaches crystal scoring techniques are presently under development. We designed and developed a reliable approach combining latest multi-channel in situ DLS techniques and micro-pump technology to score and sort in high throughput mode crystal suspensions for SFX applications. The present sorting hardware and microfluidics carrier is designed to be combined also with other detection methods, e.g. second harmonic generation (SHG); (confocal) depolarized dynamic light scattering (DDLS) and UV light fluorescence detection. Details will be presented.

Keywords: Crystal Sorting, XFEL, SFX, DLS 